\title{
Cerebro, pensamiento y comunicación: un acercamiento a las formulaciones de Rubén Arboleda Toro
}

\author{
Danilo José de la Hoz Páez ${ }^{1}$ \\ Universidad de Cartagena
}

\begin{abstract}
Resumen
Este trabajo expone las formulaciones del investigador colombiano Rubén Arboleda Toro sobre la sintaxis, formulaciones que han nacido del ejercicio intelectual de repensar la sintaxis en el marco de una teoría del conocimiento.
\end{abstract}

Palabras clave: realidad, cerebro, pensamiento, comunicación, representaciones mentales.

\begin{abstract}
This paper recovers some reflexions on syntax, in which the central proposition has been elaborated by Rubén Arboleda Toro. Professor Arboleda's formulations have arisen from the intellectual exercise of rethinking syntax in the frame of a theory of Knowledge.
\end{abstract}

Key Words: Reality, Brain, Thinking, communication, mental representations.

\section{Introducción}

Este documento recupera reflexiones sobre sintaxis, cuya propuesta central fue elaborada por Rubén Arboleda $\mathrm{Toro}^{2}$ en el Seminario Teórico I de la Maestría en Lingüística (Convenio Universidad Nacional

\footnotetext{
${ }^{1}$ Profesional en Lingüística y Literatura de la Universidad de Cartagena y candidato a Magíster en Lingüística de la Universidad Nacional de Colombia. Actualmente se desempeña como docente de la Universidad de Cartagena y la Universidad Tecnológica de Bolívar. Miembro del Grupo de Investigación TEXCULTURA (Universidad de Cartagena-Universidad de Córodoba-Universidad del Altántico). email: tenorlirico75@gmail.com

${ }^{2}$ Docente-Investigador del Departamento de Lingüística de la Universidad Nacional deColombia. Estudios de maestría en Lingüística Hispánica del Instituto Caro y Cuero, Seminario Andrés Bello, y de doctorado en Lingüística Hispánica de la Universidad Autónoma de México. Ha participado en el desarrollo de la programación lingüística del Instituto Caro y Cuervo y su unidad docente, el Seminario Andrés Bello, así como de algunas universidad de la Amazonía y Córdoba. Actualmente trabaja en el libro De la sílaba al texto: temas de lingüística general y española.
} 
de Colombia-Universidad de Cartagena), desarrollado en la ciudad de Cartagena de Indias en octubre de 2007. Las formulaciones del profesor Arboleda han nacido del ejercicio intelectual de repensar la sintaxis en el marco de una teoría del conocimiento.

\section{Cerebro, pensamiento y comunicación}

La cultura occidental heredó de la filosofía platónica y de la tradición cristiana una concepción de ser humano escindido, dividido en dos dimensiones contrapuestas: alma/cuerpo, donde lo espiritual ocupa un lugar preponderante sobre lo corporal; y heredó de Descartes la dicotomía: mente/cuerpo. Con base en recientes descubrimientos neurológicos, sin embargo, el profesor Rubén Arboleda Toro dignifica lo corpóreo al concebir al pensamiento como una realidad biológica. Este nuevo punto de vista nos aproxima a una concepción de ser humano que armoniza los polos contrapuestos antaño y, por lo tanto, ofrece una mirada mucho más integral.

El profesor Arboleda concibe el cerebro humano como un órgano biológicamente capacitado para $a$ ) construir conocimientos y $b$ ) para comunicarlos. Por un lado, la construcción de conocimientos implica unas fuentes, unos insumos, un proceso y un producto:

a. Fuentes: el ser humano construye conocimientos que provienen de las percepciones sensoriales, del carácter autogenerativo del cerebro y por actos predicativos de otros.

b. Insumos: extramentales (las realidades en sus relaciones y las señales de los actos predicativos) y mentales (representaciones previas).

c. Proceso: la construcción de conocimientos se lleva a cabo a través de varias funciones y procesos cerebrales innatos, tales como: representación cerebral, memorización, contrastación, reconocimiento, generalización y clasificación.

d. Producto: significado y su sintaxis (esto es, conocimiento, representaciones mentales).

La comunicación, por su parte, implica la simbolización y la convencionalización. Al respecto, el profesor Arboleda considera que el cerebro humano, merced a una conquista evolutiva, está capacitado para comunicar los conocimientos que construye. A continuación profundizaremos en los planteamientos del profesor Arboleda y los complementaremos con algunas reflexiones y propuestas personales. 


\section{Representaciones sensoriales}

El profesor Arboleda considera que, en primera instancia, el cerebro humano construye ciertos conocimientos mediante la representación de las realidades auditivas, gustativas, olfativas, táctiles y visuales que impresionan los sentidos. De lo anterior se infiere que estas representacionessensoriales constituyenconocimientosno-verbales, es decir, que no implican la palabra, el signo verbal. El ser humano se representa objetos de la realidad (entiéndase seres humanos y no humanos), eventos que suceden en los objetos (desplazamiento, movimientos inmanentes como el crecimiento, etc.), eventos en los que participan objetos (acción ejercida por un objeto sobre otro, incluido el ser humano mismo, etc.), propiedades de objetos y eventos, ubicación de objetos y eventos en el espacio y en el tiempo y grado con que los objetos y eventos poseen propiedades que en ellos se perciben.

El cerebro se representa las realidades que impresionan los sentidos y sus relaciones, archiva las representaciones (las memoriza), reconoce las realidades cuando vuelven a impresionar, y establece clasificaciones. En el proceso de reconocimiento el cerebro contrasta la realidadimpresionadora conlas representacionesmemorizadashasta encontrar la que le corresponde, pero si la realidad impresionadora no se reconoce, entonces el cerebro la memoriza. Es importante anotar que todos estos procesos de representación cerebral, memorización, contrastación, reconocimiento, generalización y clasificación son dimensiones del conocimiento y el pensamiento que no se aprenden. Alcanzada la maduración correspondiente, el cerebro conoce y piensa en esa medida, así como, alcanzada la maduración, el ojo ve, el aparato digestivo digiere, el aparato respiratorio respira y el aparato reproductor produce óvulos y espermatozoides. Es decir, es una disposición instintiva, funciona al margen de la voluntad, constituye una acción cerebral biológicamente inevitable.

Aunque el profesor Arboleda no define explícitamente el concepto de "representación", podemos entender este concepto, desde la lectura de su texto y sus explicaciones, en dos sentidos:

a) Representación como 'función'. El Diccionario de la Real Academia Española (DRAE, 2007), define el término "función" así: "(Del lat. functǐo, -ōnis). f. Capacidad de actuar propia de los seres vivos y de sus órganos, y de las máquinas o instrumentos". Precisamente, Arboleda (2007) caracteriza la representación como una función instintiva del cerebro y, por lo tanto, biológicamente inevitable: "El humano tiene un 
cerebro que se representa las realidades que impresionan los sentidos, archiva las representaciones (las memoriza), reconoce las realidades cuando vuelven a impresionar y establece clasificaciones. En el cerebro humano se realizan todas estas funciones instintivamente... (es decir)... al margen de la voluntad" (Las cursivas son nuestras). El autor enfatiza su caracterización estableciendo una analogía entre la función digestiva y la representación, como vimos arriba.

b) Representación como "proceso". El DRAE (2007) define el término "proceso" así: "(Del lat. processus). m. Conjunto de las fases sucesivas de un fenómeno natural o de una operación artificial". Al respecto, de algunos apartes del texto de Arboleda podemos deducir la acepción de representación como proceso, en cuanto implica fases sucesivas, como veremos en el siguiente aparte.

c) Representación como "producto". El profesor Arboleda sostiene que la representación de un objeto no es el objeto. Insinúa que la representación es un "producto" al afirmar que la representación es "significado" y, por lo tanto, "conocimiento" (fijémonos en que ambos significantes remiten a entidades, objetos), pero no se compromete con una definición explícita (¿Qué son esas entidades? ¿Impulsos nerviosos? ¿Huellas de los impulsos? ¿Imágenes?...). Ofrece, no obstante, algunas características muy esclarecedoras: 1) las representaciones tienen capacidad de adaptación, lo cual permite el ajuste del conocimiento, del significado: "Una realidad ya representada es susceptible de experimentar cambios; también sucede que la persona sea impresionada por elementos de una realidad que no la habían impresionado en una primera ocasión" (2007). Podría pensarse que esta capacidad adaptativa es plástica en la medida en que dos representaciones de una misma realidad pueden coexistir o integrarse; 2) las representaciones tienen un carácter autogenerativo, es decir, que el cerebro puede procesar nuevas representaciones (denaturaleza estrictamente mental) a partir de representaciones previas que emanan de experiencias sensoriales inmediatas.

Además de ser un fenómeno natural e instintivo, en Arboleda la construcción del conocimiento sensorial implica un proceso, es decir, un conjunto de fases sucesivas. Al respecto, en este artículo proponemos la siguiente hipótesis: cuando nos referimos a la representación sensorial de realidades, debemos tener en cuenta 
que ésta consta de, por lo menos, dos fases: análisis y síntesis. A continuación, explicaremos este planteamiento.

\section{a) Nivel básico de representación sensorial (Análisis)}

Cuando el cuerpo de un ser humano es estimulado por una realidad específica, cada uno de sus sentidos realiza un proceso de percepción analítica de dicha realidad. Ese proceso analítico consiste en la descomposición física (ojo, oído, piel) y/o química (gusto, olfato y piel) de la realidad, que desencadena la fabricación cerebral de un constructo particular ("representación sensorial individual") para cada una de las percepciones sensoriales de la realidad extramental en cuestión y de sus relaciones con otras realidades. Ese constructo se memoriza y queda a disposición de futuras activaciones y modificaciones. Por ejemplo, al percibir visualmente la realidad "mango maduro" como una entidad extramental con una masa que ocupa un espacio en el universo, el cerebro representa su "figura" y percibe su color "amarillo", y, simultáneamente, a través de los otros sentidos, percibe otras realidades consustanciales a aquélla: gracias al sentido del gusto, percibe el sabor "dulce" y la textura "fibrosa", y gracias al sentido del olfato, percibe el olor "fragancia [de mango]"3, entre otros. El objeto "mango maduro" es una totalidad en el universo (extramental), pero en la mente se halla descompuesto en representaciones específicas de cada una de las realidades consustanciales a éste (sus "propiedades"), percibidas (mediante análisis) por cada uno de nuestros cinco sentidos: "figura", "amarillo", "dulce”, "fibroso", "fragancia (de mango)", etc.

\section{b) Nivel secundario de representación sensorial (Síntesis)}

Aunque el cerebro, en primera instancia, se representa las realidades descompuestas, esto no implica que en él las representaciones reposen desarticuladas, sin conexión alguna, puesto que el cerebro, además de la representación particular de cada una de las realidades consustanciales (partes) de una realidad (todo), simultáneamente se representa las relaciones entre éstas. Por ello, las representaciones sensoriales básicas son tejidas en una red que reconstruye, virtualmente, la totalidad descompuesta durante el análisis (síntesis).

Cuando el cerebro, por ejemplo, es estimulado con la señal /mango maduro/ o por el mismo objeto extramental "mango maduro", se activa la red de relaciones entre las representaciones sensoriales básicas,

${ }^{3}$ Dado que no existe una señal específica con la cual significar ese olor, usamos esta limitada convención. 
reconstruyéndose así la totalidad (síntesis). En otras palabras, no sólo se activa la representación de una "figura", sino que, junto con ésta, se activa (simultáneamente) la representación de "amarillo", la de "dulce", la de "fragancia", etc., y todas juntas constituyen un nivel de significado más complejo que el anterior, pues sumadas conforman un objeto diferente a ellas mismas. Haciendo una analogía, diríamos que la red de representaciones sensoriales básicas funciona, no tanto como un "rompecabezas", pues en éste las fichas tienen un lugar fijo y predeterminado por la figura total, sino que más bien esa red funciona como un "armatodo", donde las piezas pueden ensamblarse de diversos modos para obtener así diversos tipos de figuras. Por ejemplo, con la red de representaciones conformada por "amarillo", "dulce", "fragancia [de mango]", entre otros, podemos construir diversas representaciones secundarias como "amarillo mango", "mango amarillo", "fragancia de mango", "mango fragante", "dulce de mango", "mango dulce", etc.

En la red representacional básica el cerebro puede hacer primar una representación por encima de las demás, y hacerla funcionar como núcleo en torno a la cual giren las otras. Ser núcleo de una representación es un rol, una función que puede ser realizada por cualquiera de las representaciones básicas que conforman la red. En esta medida consideramos que la sintaxis no se circunscribirá únicamente a las relaciones entre los objetos-todo reconstruidos en las redes representacionales que llamamos secundarias (2), sino que hay una sintaxis en el nivel básico representacional (1), por cuanto las representaciones sensoriales más elementales se relacionan entre sí como partes de un todo, con el fin de reconstruir virtualmente en la mente un objeto extramental. En cada una de esas redes básicas hay un núcleo que determina la relación entre las representaciones que hacen parte de cada red. Es decir, que allí también existen relaciones de atribución: hay determinantes y determinados.

Si examinamos los ejemplos anteriores nos percataremos de que en "amarillo mango" y "fragancia de mango", los núcleos respectivos son "amarillo" y "fragancia", respectivamente, y todas las otras representaciones de la red se subordinan a éstas. De lo anterior, se infiere que el proceso de análisis sensorial que llevan a cabo nuestros sentidos sobre los objetos, determina (es directamente proporcional) el proceso de representación analítica que realiza nuestro cerebro de dichas realidades: el cerebro es capaz de percibir un objeto extramental como una totalidad, en la medida en que, primero, 
percibe y representa sus propiedades como objetos en sí mismos (análisis) y, luego, los relaciona (síntesis) mediante una red ${ }^{4}$.

\section{Representaciones, fruto de la arbitrariedad de la mente}

Como se dijo al inicio, el profesor Arboleda plantea que, además de que existen objetos que son fruto de la representación (representaciones sensoriales), hay objetos que son fruto de la arbitrariedad de la mente (representaciones conceptuales). Respecto al último tipo de objetos aclara que pueden tener naturaleza distinta: hay algunos que se construyen con base en representaciones sensoriales previas, mientras que existen otros que se construyen con base en conceptos abstraídos o a actos predicativos de otros. De acuerdo con Arboleda, la representación autogenerada consiste en generar, por abstracción, la representación de una realidad mental sobre la base del conocimiento sensorial previo. Estas realidades mentales o imaginarias llegan a ser representadas fuera de la mente mediante dibujos, maquetas, esculturas... o mediante la descripción verbal.

Por ejemplo, el objeto mental sirena se genera al descomponer las representaciones mujer y pez en dos partes (superior/inferior), privilegiar una de las partes en cada representación (tronco/cola) y unir las partes privilegiadas. Este nivel es el que permite explicar -en parte- el origen y la dinámica de los procesos creativos en el ser humano y los juegos de la imaginación. Aquí encuentran su génesis objetos mentales como Cíclope, Superman o Alien. Al respecto, consideramos que, a pesar de las diversas naturalezas de las representaciones conceptuales, en la construcción de éstas el cerebro echa mano de los mecanismos de análisis/síntesis y contrastación/ generalización. Veamos:

\footnotetext{
${ }^{4}$ Por ello considero que las propiedades (por lo menos las de origen sensorial), en cuanto son en primera instancia sustancia del contenido, son objetos en sí mismos: "objetos contenidos" que conforman un "objeto contenedor". Disiento de Arboleda cuando sostiene que la mente transforma las propiedades en objetos, al prescindir cognitivamente del objeto al cual pertenece una propiedad. Se trata de todo lo contrario: las propiedades son, en primera instancia, percibidas y representadas como objetos, y por ello es que el cerebro las puede tratar como objetos; y, en segunda instancia, es que son consideradas como propiedades, en la medida en que se consideran como partes que conforman (dependen de) un todo (es en este segundo nivel donde se consideran sus relaciones de atribución con el 'objeto todo'). Los humanos hemos convencionalizado signos adjetivos para significar los "objetos contenidos" asociados a "objetos continentes" (propiedades), y signos sustantivos para significar a los "objetos continentes" (objetos propiamente dichos). Pero cuando nos referimos a los primeros como realidad individual (no asociados a "objetos continentes") los significamos con signos sustantivos.
} 


\section{a) Nivel básico de representación conceptual (Contrastación y Generalización)}

Ocurre cuando el cerebro, por operaciones de comparación y contraste entre diversas redes de representaciones sensoriales individuales hace caso omiso de las representaciones diferentes que conforman las redes y privilegia la similitud. Por ejemplo, cuando utilizamos un concepto como el de "árbol", implícitamente hacemos caso omiso de las distinciones entre los árboles individuales. Así, aunque existen diferencias visibles entre un roble, un olmo, un arce, un pino y una acacia, podemos hablar de ellos en términos generales, dado el común denominador de hojas, tronco, ramas, raíces, etc. El concepto "árbol", como cualquier otro, implica un privilegio de la similitud (Ejemplo tomado de Issacharoff \& Madrid, 1994: 30). El concepto es así una suerte de entidad mental que no proviene directamente de la percepción sensorial, sino que surge del cerebro mismo. Es una red sintética de representaciones similares, extraídas -por abstracción- de otras redes de representaciones sensoriales diferentes.

\section{b) Nivel secundario de representación conceptual (Contrastación y Generalización)}

Ocurre cuando el cerebro, por operaciones de comparación y contraste entre diversas representaciones conceptuales básicas con base en su similitud, emana una representación conceptual mucho más general. Por ejemplo, el concepto "animado" se genera al comparar representaciones conceptuales como "humano", "animal" y "vegetal". Cabe anotar que la representación conceptual puede extenderse a niveles terciarios, e incluso podríamos pensar en mayores niveles de abstracción. Pero, de todos modos, la dinámica es la misma que la del nivel secundario. Además de la representación de conceptos, el cerebro tiene la capacidad de fabricar objetos sin existencia previa en la dimensión extramental, como veremos a continuación:

\section{Representación por actos de predicación}

Por último, Arboleda plantea que el cerebro puede construir una representación de objeto, no con base a la realidad sensorial o en el carácter autogenerativo, sino en las instrucciones precisas que otro hablante nos da por medio de señales verbales. Al respecto, el autor sostiene que la predicación es el gran descubrimiento del humano en su evolución. Es una práctica esencialmente humana que nace de la necesidad de compartir un conocimiento con otro que no lo tiene, $y$, por lo tanto, fue construida en respuesta a esa necesidad (hipótesis filogenética). El humano experimenta que 
no puede transferir directamente su conocimiento a otros (no es telepático), pero descubre que la predicación puede activar en otros el mismo conocimiento que se está pensando y/o desencadenar la construcción de una nueva representación en relación con un objeto de atribución.

Por ejemplo, los zoólogos pueden activar en nuestra mente el objeto "ornitorrinco" por medio de una descripción ("es un mamífero pequeño como una rata, con el cuerpo cubierto de pelo, pico y patas semejantes a las de un pato, que pone huevos y vive a las orillas de los ríos"), aunque nunca hayamos visto tal objeto. Como podemos ver, la construcción de este tipo de representaciones consta de las mismas fases de la construcción de representaciones autogeneradas: análisis y síntesis. Fijémonos en que la descripción (constituida por señales) produce la activación de representaciones previas que se privilegian (análisis) y se enlazan en una nueva red (síntesis). Al respecto, Cuenca y Hilferty (1999: 32) sostienen que el cerebro humano está potenciado genéticamente para llevar a cabo dos procedimientos: la generalización o abstracción y el análisis o discriminación. Explican que generalizar es obviar las diferencias entre realidades y agruparlas según sus semejanzas, mientras que analizar es insistir en los rasgos diferenciales de dos o más realidades con la finalidad de no confundirlas entre sí. Los autores aclaran que si no generalizáramos no podríamos sobrepasar el nivel de las entidades individuales y la realidad sería caótica y constantemente nueva, de forma que no podríamos llegar a una estructuración conceptual. Y si no analizáramos, todo sería uno y tampoco habría pensamiento complejo.

Hasta aquí hemos visto que, de acuerdo con Arboleda, el humano construye conocimientos que provienen de las percepciones sensoriales, del carácter autogenerativo del cerebro y por actos predicativos de otros. A continuación, veremos otro tipo de representación: la relación que existe entre las realidades.

\section{La sintaxis como significado y el significante de la sintaxis}

Por otro lado, de acuerdo con Arboleda, las realidades no se representan aisladas. El ser humano, junto con las realidades, se representa las relaciones que existen entre éstas. De allí concluye que la sintaxis no es sólo un asunto de significantes, puesto que es "significado", en la medida en que es una representación de relaciones de presuposición entre las representaciones autónomas. 
Las realidades se representan en el cerebro humano como objetos, eventos que suceden en los objetos (desplazamiento, movimientos inmanentes como el crecimiento, etc.), eventos en los que participan los objetos (acción ejercida por un objeto sobre otro, incluido el humano mismo, etc.), propiedades de objetos y eventos, ubicación en el espacio y en el tiempo, y grado con que los objetos y eventos poseen las propiedades que en ellos se perciben.

Pero las realidades no se representan aisladas... Se configura algo que podríamos considerar como percepción y representación de orden atribucional (Arboleda, 2007. "La sintaxis como significado y el significante de la sintaxis")

En este pasaje "representación de orden atribucional" se entiende como "Principio de rección", "régimen" o "relación de presuposición". Es un asunto de redes neuronales que se desencadenan, esto es: una propiedad presupone un objeto (pues las propiedades se perciben en los objetos); un evento presupone unos participantes (porque los eventos se perciben como realidades en las que intervienen objetos); en los eventos los objetos se perciben como agentes o como pacientes y, por lo tanto, en los eventos se perciben relaciones entre objetos.

Así pues, además de la representación de los diferentes aspectos de la realidad, en la mente de los humanos se representan relaciones entre distintos aspectos de ella. Si la representación es conocimiento, los humanos alcanzamos conocimiento de distintos aspectos de la realidad y conocimiento de sus relaciones. El conocimiento humano no es entonces de elementos aislados, sino de elementos en relación. Es decir, el conocimiento humano es relacional, sintáctico, en ese sentido. Hay en el conocimiento una sintaxis: la sintaxis del conocimiento, que también es conocimiento. Y esa sintaxis del conocimiento humano deviene de la sintaxis del mundo. Pero no sólo de ahí, sino también de la manera cómo el humano percibe el mundo, de las relaciones que establece, de la manera cómo lo organiza. Es en el pensamiento y el conocimiento humanos donde las relaciones entre los elementos del mundo conocido y pensado adquieren el carácter atribucional. La atribución es una actividad de pensamiento.

Así como necesitamos simbolizar las representaciones autónomas, necesitamos simbolizar (significante sintáctico) las relaciones entre las representaciones (sintaxis del conocimiento). En el significante sintáctico se hallan las variaciones en la manera de significar la sintaxis de la representación (diferencias entre las lenguas). La 
representación de una realidad dada conlleva el aprendizaje de relaciones de esa realidad con otras, esto es, el aprendizaje de esa realidad conlleva el aprendizaje de su sintaxis. El conocimiento del signo léxico de esa realidad conlleva el aprendizaje de relaciones posibles de ese signo con signos de otras realidades. Es decir, conlleva el conocimiento de su sintaxis. La activación de un signo en el cerebro de los hablantes conlleva la activación de sus posibilidades relacionales o combinatorias (esto es, su significado sintáctico). El conocimiento del significado de los signos, incluido el conocimiento de sus posibilidades relacionales, es factor decisivo para que, a partir de la activación de un signo en el cerebro de una persona, se generen redes sígnicas atribucionales (redes sígnicas sintactizadas) y para que en un hablante se establezcan las relaciones correspondientes entre los signos constitutivos de un determinado texto que alguien le dirija.

Desde esta perspectiva, el vocabulario (inventario de símbolos de objetos, eventos, propiedades y grados) es una unidad sintáctica, ya que las reglas sintácticas no son ajenas al vocabulario; la regla no es una realidad externa al vocabulario. Desde el punto de vista atribucional, las reglas serían una formulación de las regularidades relacionales de los signos. El vocabulario es una unidad sintáctica porque consta de la representación de objetos y sus relaciones, más su significante: el signo léxico significa (indica) él mismo la representación de una determinada realidad con sus relaciones posibles (con su sintaxis). Pero existen contextos donde el léxico no es suficiente para indicar la conexión pretendida por una persona (como veremos luego), debido, por ejemplo, a que el texto contenga varios signos con los cuales otro signo dado pueda establecer conexión (dirección de la relación sintáctica). Por ello, los hablantes de lenguas han recurrido a mecanismos auxiliares para marcar dicha dirección. Estos mecanismos conforman un sistema de índices de dirección ${ }^{5}$ auxiliares del vocabulario, entre los que se encuentran: el orden, la concordancia, las partículas y la entonación (no indican la relación, lo que señalan es el constituyente con el cual se establece la relación: la dirección de la relación). De aquí, podemos concluir que las relaciones entre las realidades representadas en la mente se significan en las lenguas por medio del vocabulario y por medio de los demás mecanismos indicadores de la dirección de la relación significada.

\footnotetext{
${ }^{5}$ Nos parece más precisa la denominación indicadores de dependencia, puesto que la dirección es un valor que hace parte del concepto de dependencia (véase Simone, 2000: 160 y ss.).
} 


\section{Representación de objetos y las categorías gramaticales de los nombres}

De acuerdo con Arboleda, el ser humano percibe y se representa objetos, eventos, propiedades de objetos y eventos y grados de propiedad. Respecto a los objetos, cabe preguntarnos: ¿qué es lo que nuestros sentidos y cerebro perciben y se representan de los objetos? Tomando en consideración las señales convencionalizadas por las lenguas del mundo para activar las representaciones de objetos de sus hablantes, podemos inferir que las personas percibimos y representamos, entre otros, los siguientes rasgos inherentes a los objetos:

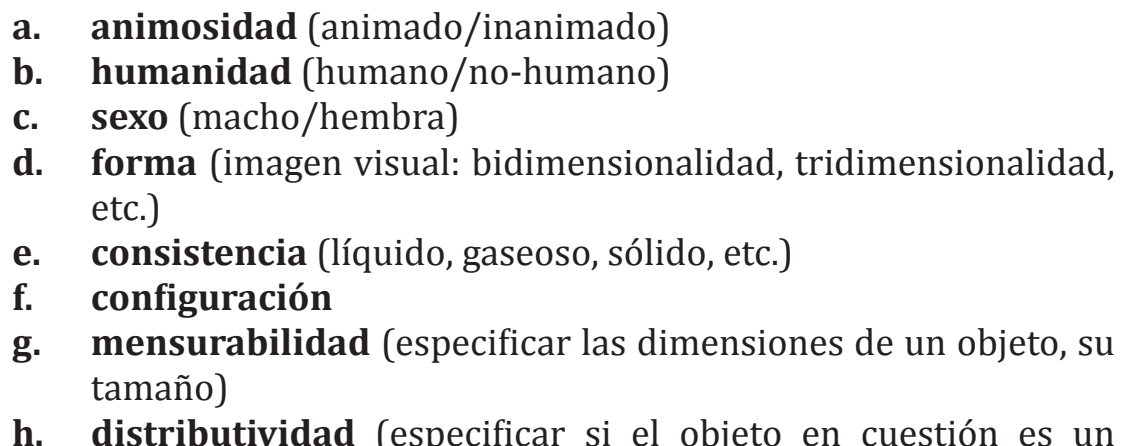
todo concentrado en un lugar particular o si es un conjunto de unidades distribuidas en un espacio)

i. colectividad (oponer un objeto a un conjunto)

j. rol o función

k. localización espacio-temporal

Las lenguas han convencionalizado señales lingüísticas como la clasificación nominal (género o clase nominal; clasificador), la cuantificación (numérica y no-numérica), la referencialidad (definido/indefinido), la deixis (personal y espacial) y los casos (directos y oblicuos) para marcar los signos sustantivos y adjetivos (los nombres). El propósito es tanto cognitivo como comunicativo. Desde el punto de vista semántico, cognitivo y cultural, la función de todos ellos es significar los paradigmas en los que se clasifican los objetos, dada la manera como interactuamos con ellos. Desde el punto de vista comunicativo, el propósito de las marcas es establecer la concordancia entre los signos sustantivos y el resto del enunciado y/o cumplir una función pragmática. 


\section{La representación de eventos y las categorías gramaticales de los verbos}

En relación con los eventos, el humano es capaz de representarse no sólo los eventos y sus participantes, sino que junto con éstos se representa su ubicación en el continuum del tiempo real, con el fin de especificarlos, caracterizarlos. Para llevar a cabo esa ubicación temporal, el humano toma como punto de referencia un momento del tiempo, y ese punto o intervalo de tiempo privilegiado es denominado por los lingüistas locus temporal. Aunque el locus temporal puede ser cualquier punto en la dimensión temporal, la mayoría de los hablantes da prioridad al locus temporal definido por el momento en que piensa o enuncia un texto/discurso: locus absoluto. Si el locus temporal es definido por otro momento (ayer, entonces, etc.) se llama locus relativo (Véase Simone, 2001: 270-4).

La razón principal para que los hablantes demos prioridad al locus temporal absoluto (definido por el momento en el que pensamos o enunciamos un discurso), es porque el pensamiento tiene carácter corpóreo, es decir, se basa en la experiencia corporal humana. Es lo que se conoce como naturaleza corpórea o corporeización del lenguaje: el núcleo de nuestros sistemas conceptuales se basa directamente en nuestra percepción de la realidad, en el movimiento corporal y en la experiencia física y social (Lakoff y Johnson, 1986). El intervalo de tiempo del evento puede ser anterior al locus temporal (pasado), puede ser simultáneo al locus temporal (presente) o puede ser posterior (futuro). Hay hablantes que han convenido marcar en su lengua la división tripartita. Pero hay otros hablantes que han convenido marcar en su lengua sólo dos divisiones (pasado/no pasado, futuro/no futuro, presente/ no presente).

Por ejemplo:

\section{(1) Lituano 6}

$\begin{array}{lll}\text { dirb-au } & \text { dirb-u } & \text { dirb-siu } \\ \text { trabajar-1S + PASADO } & \text { trabajar-1S + PRESENTE } & \text { trabajar-1S + FUTURO } \\ \text { "Trabajé, estaba trabajando" } & \text { "Trabajo, estoy trabajando" } & \text { "Trabajaré, estaré trabajando" }\end{array}$

\footnotetext{
${ }^{6}$ Tomado de Chung y Timberlake (1985: 204-5). En los ejemplos, los números "1", "2", y "3" significan primera, segunda y tercera persona, mientras las letras "S" y "PL" significan singular y plural, respectivamente. Así, "1S" debe leerse "primera persona plural”. El guión (-) debe interpretarse como unión entre morfemas. Y los textos entre comillas (“...") deben considerarse, no como una traducción, sino como una "aproximación" en nuestra lengua española a lo que podría significar el enunciado de la lengua objeto de análisis.
} 


\title{
(2) Takelma (Estados Unidos)
}

$\begin{array}{ll}\text { yaná-t'ē } & \text { yãn-t'e } \\ \text { ir-1S + FUTURO } & \text { ir-1S + NO FUTURO } \\ \text { "iré" } & \text { "fui, estoy yendo, estoy a punto de ir" }\end{array}$

\section{(3) Yidiñ (Australia)}

\author{
nayu gundii? \\ yayu gundi?-ala \\ $1 \mathrm{~S}+$ regresar + PASADO \\ $1 \mathrm{~S}+$ regresar + NO PASADO-ahora \\ "regresé" \\ "estoy regresando, estoy a punto de regresar"
}

Además, el humano -mediante un ejercicio analítico que lleva a cabo su cerebro- es capaz de percibir la constitución temporal interna de un evento: es decir, su cerebro es capaz de objetivar un evento y descomponerlo en etapas o fases (inicio, desarrollo y finalización) con el objetivo de alcanzar un conocimiento más profundo de éste. $\mathrm{Y}$ ese nivel de detalle permite al humano percibir si el evento se cumplió a cabalidad, si se está llevando a cabo, o si está inconcluso, entre otros. A esta representación de la constitución temporal interna de un evento los lingüistas la han denominado aspecto (Cf. Simone 2001: 274-8).

La constitución temporal interna de un evento es percibida y representada por los humanos en términos de:

- Su completud: si el evento se ha cumplido (perfectivo) o si aún no se ha cumplido (imperfectivo).

- La fase de desarrollo del evento: si el evento está apenas iniciando (incoativo) o si ya finalizó (cesativo o terminativo).

- Si el evento se está desarrollando o no en un momento preciso: si el evento se percibe en pleno desarrollo (progresivo o continuo).

- La extensión temporal del evento: si el evento se percibe como momentáneo (puntual); si se percibe como teniendo duración y sin cambios en su desarrollo (durativo); o si el evento se percibe como teniendo lugar habitualmente (habitual).

- Su concentración o distribución en el tiempo y el espacio: si el evento se percibe como una situación que se repite en momentos sucesivos (iterativo). 
Por ejemplo ${ }^{7}$ :

\section{(4) Húngaro}

a levelet írom
la carta escribir
"escribo la carta"

\section{(5) Ruso}

$\begin{array}{ll}\text { Gor } & \text { "quemarse" } \\ \text { cves "florecer" za-cvet } \\ \text { kur "fumar" }\end{array}$

\section{6) Bafia}

PUNTUAL

àkàn-? "él escribe"

HABITUAL

ákàn-gà? "él suele escribir" a levelet meg-írom

la carta IMPERFECTIVO-escribir

"estoy completando la escritura de la carta"

\section{(7) Yujup}

حăh wédní - bèh

1S comer-ITERATIVO

“yo como (una y otra vez)”

\section{NO PROGRESIVO}

John sings well

John sang well

"Juan canta bien"

"Juan cantó bien"

za-gor-sja "empezar a

quemarse"

"empezar a florecer" ot-cvet

"empezar a fumar" do-kur do-gor "terminar de

quemarse"

"terminar de florecer"

"terminar de fumar"
DURATIVO

ákàn-kàn "él está escribiendo"

ITERATIVO

ákànga?-kangà? "él escribe habitualmente"

John will sing well “Juan cantará bien" John will be singing well "Juan estará cantando bien"

Asimismo, el humano atribuye la representación de una intención específica a la representación de un evento, pues percibe una relación de atribución entre intenciones y eventos. Y cuando se comunica es capaz de activar esas relaciones atribucionales en otros y de reconocerlas cuando otro le dirige un texto. Para ello, los hablantes han convenido en usar algunas formas lingüísticas unidas a los

${ }^{7}$ Los primeros seis ejemplos y el octavo son tomados de Mel'cuk (1994) y el séptimo de Ospina (2002). 
significantes de representaciones de eventos como activadoras de representaciones de intenciones, las cuales han sido denominadas por los lingüistas como modo:

Indicativo: expresa una declaración que puede ser verdadera o falsa. Ej: Luisa se está comiendo el almuerzo.

Imperativo: expresa una orden y representa un acto por medio del cual el locutor manifiesta su voluntad y trata de influenciar al oyente para que realice ciertos actos. Ej: Luisa, cómete el almuerzo.

Optativo: expresa el deseo del locutor. Ej: Si Luisa se comiera el almuerzo.

Subjuntivo: expresa la afirmación del carácter no "real" del evento significado por el signo verbo, que es el objeto de otro signo verbo que significa "querer", "esperar". Ej: La mamá de Luisa quiere que se coma el almuerzo.

Condicional: expresa la afirmación de carácter condicional del evento. Ej: Luisa se comería el almuerzo si estuviera rico.

Irreal: expresa una hipótesis contraria a la realidad. Ej: Si Luisa se hubiera comido el almuerzo, su mamá estaría feliz.

Los hablantes también perciben una relación atribucional entre los eventos y ciertas actitudes de los hablantes hacia los eventos. Por ello, han convenido en unir a los signos verbales propios de las representaciones de eventos algunos signos que significan actitudes con respecto a lo que piensan y dicen del evento. El hablante puede percibir, representar, pensar y expresar su actitud respecto a:

- La necesidad, la posibilidad o imposibilidad de que un evento se realice (modalidad alética).

- La obligación, el poder, el deseo de que suceda un evento (modalidad deóntica).

- El compromiso del hablante con su certeza o duda con respecto a lo que está pensando o diciendo de un evento (modalidad epistémica).

- La fuente de la información del evento (modalidad evidencial): el locutor ha percibido directamente el evento que describe (experiencial), el locutor describe un evento que le ha sido contado por otra persona (citativo), el locutor infiere un evento a partir de ciertos eventos relacionados (inferencial), el locutor usa sus conocimientos previos para suponer un evento (presuntivo). 
Por ejemplo ${ }^{8}$ :

\section{(9) Yujup (Makú Puinave)}

ăh àktígn ùh ùbmú

1S semilla de yuca POSIBILIDAD sembrar

"Yo posiblemente sembraré semilla de yuca"

$$
\text { ăh àktígn ùbmú ùh }
$$

1S semilla de yuca sembrar POSIBILIDAD

"Yo sembraré semilla de yuca, posiblemente"

\section{(10) U'wa (Chibcha)}

sisbura jajro eb wihk-ta-ro

gallina hambre maíz dar-NECESIDAD-DECLARATIVO

"Las gallinas tienen hambre, hay que darles maíz"

\section{(11) Japonés}

$\begin{array}{lll} & & \\ & & \text { DESIDERATIVO } \\ \text { nom-u } & \text { "beber" } & \text { nomi-tai "querer beber" } \\ \text { mi-ru } & \text { "ver" } & \text { mi-tai "querer ver" } \\ \text { wakar-u "comprender" } & \text { wakari-tai "querer comprender" }\end{array}$

\section{(12) Húngaro}

$\begin{array}{lll} & & \text { POTENCIAL } \\ \text { Készit "preparar" } & \text { készit-het "poder preparar" } \\ \text { Jár } & \text { "caminar" jár-hat } & \text { "poder caminar" } \\ & & \text { DEBITATIVO } \\ \text { Seda } & \text { "coser" } & \text { seda-bcu "tener que coser" } \\ \text { Nate } & \text { "esperar" } & \text { nate-bcu "tener que esperar" }\end{array}$

\section{(13) Yujup (Makú Puinave)}

ìhw dédní bàh
Ihow venir CITATIVO
"Dizque Ihow viene"

\author{
〜ìdh àbmá hó \\ 3PL bailar INFERENCIAL 2 \\ "Parece (por el ruido) \\ que están bailando"
}

Las lenguas cuentan con herramientas simbólicas para significar la representación de la ubicación temporal de los eventos y sus participantes, de la constitución temporal interna de los eventos, y de las intenciones y de las actitudes ligadas a los eventos. Algunas lenguas lo hacen mediante signos mínimos (morfemas) o mediante signos léxicos (palabras). Es importante aquí anotar que, al involucrar el cuerpo como foco central de la experiencia, se puede explicar con mayor facilidad el fenómeno de la intercomprensión entre las

${ }^{8}$ El ejemplo (9) y (13) es tomado de Ospina (2002); el (10) de Casilimas (2003); y (11) y (12) de Mel'cuk (1994:179 y 1994: 180-1, respectivamente). 
personas a pesar de las diferencias superficiales en las lenguas, puesto que los puntos en común son mayores que las diferencias. Marcar una división temporal u otra es sólo un accidente. Ello no significa que los hablantes perciban una realidad temporal distinta. Usar signos mínimos (morfemas) o signos léxicos (palabras), es sólo una diferencia superflua. Al estar dotados con los mismos sentidos $y$, por ende, con las mismas posibilidades perceptivas ${ }^{9}$ (las mismas ventanas hacia el universo), los humanos estamos destinados a percibir y representar los mismos objetos, eventos, propiedades y grados de propiedades, y las mismas relaciones entre tales realidades.

\section{Conclusión}

En primer lugar, podemos afirmar que la propuesta teórica del profesor Rubén Arboleda Toro es dialógica, puesto que en ella las nociones de sintaxis y semántica -consideradas de modo desarticulado por el episteme clásica de la lingüística- se funden. La sintaxis, como hemos visto, no es sólo un asunto de significantes, puesto que es "significado", en la medida en que es una representación mental de relaciones de presuposición entre las representaciones mentales autónomas. Merced a este fundamento teórico, las fronteras entre sintaxis y semántica se difuminan en un continuum, y con el término continuum no se apunta a una simple relación de complementariedad e interdependencia, sino que manifiesta una nueva visión integradora.

Podemos afirmar que la propuesta es hologramática, por cuanto pretende articular algunos sectores del conocimiento acerca del lenguaje y de la lengua. Dada la naturaleza compleja de la lengua y el lenguaje como objetos de estudio, la propuesta de Arboleda propende por la articulación de los distintos niveles (fonéticofonológico, sintáctico, semántico y pragmático) y por difuminar las fronteras entre las llamadas lingüísticas del lenguaje, de la lengua y del habla. Es decir, asume el objeto de estudio de la lingüística como

\footnotetext{
${ }^{9}$ Al respecto, en semiótica se proponen los conceptos de bauplan (Sistema de modelización preexistente que permite interpretar el mundo de los seres, objetos y acontecimientos de forma biológicamente programada), objetificación (Construcción semiósico-perceptual de objetos pertinentes que ejecutan las especies, mediante el recorte de segmentos específicos en el continuo-discontinuo físico-energético preyacente del ambiente, para el establecimiento y mantenimiento de su respectivo umwelt) y umwelt ("mundo objetivo" de cada especie vivido como "real". Es el producto complejo de la suma, esto es, de múltiples interacciones, de las objetificaciones que una especie hace de su ambiente).
} 
un todo unitario y, con esto, un carácter de complementariedad entre el todo y las partes.

Los planteamientos del profesor Arboleda parten de la configuración del significado como concepto conectivo de las distintas dimensiones y disciplinas lingüísticas para determinar los procesos psico-sociolingüísticos que involucra. En esta propuesta teórica se asume que los significados (y el sentido) son, por un lado, constructos intraorgánicos y, por otro, constructos interorgánicos. Es decir, en esta propuesta lo intraorgánico y lo interorgánico se definen mutuamente, ya que los significados construidos por el cerebro emergen de la relación del individuo con su ambiente. De aquí que cada una de las disciplinas lingüísticas que constituyen parte esencial de esta propuesta tienen que ver de algún modo con el significado.

De todo lo dicho hasta el momento, se deduce que, dadas sus características, esta propuesta de investigación podría inscribirse en el marco epistemológico del pensamiento complejo. Pensamos que, precisamente, el punto de vista cognitivo que subyace en la propuesta favorece su inserción en la lingüística del lenguaje; lo que, en consecuencia, impone la necesidad de asumir el objeto de estudio de la lingüística en su integralidad y complejidad.

\section{Bibliografía}

Arboleda Toro, Rubén. (2007). De la sílaba al texto: temas de lingüística general y española. Manuscrito no publicado(paper).

Casilimas, Clara Inés. (2003). Expresión de la modalidad en Uwa (Ponencia presentada en el X Congreso Nacional de Antropología, Manizales).

Chung, Sandra y Timberlake, Alan. (1985). Tense, Aspect and Mood en Language typology and syntactic description. Vol. III, pp 204-5

Cuenca, María Josep y Hilferty, Joseph. (1999). Introducción a la lingüística cognitiva. Barcelona: Ariel.

Diccionario de la Real Academia Española. (2007). Madrid: Real Academia de la Lengua Española.

Foley, William. (1991). The Yimas language of New Guinea. Standford: Standford University Press.

Issacharoff, Michael y Madrid, Lelia. (1994). Pensamiento y Lenguaje: el cerebro y el tiempo. Madrid: Fundamentos.

Lakoff, George y Johnson, Mark. (1986/2004). Metáforas de la vida cotidiana. 6 Ed. Madrid: Cátedra.

Mel'cuk, Igor. (1993). Cours de morphologie générale. Montréal: Presses de l'Université de Montréal, CNRS Éditions, vol. II

Ospina, Ana María. (2002). Les structures élémenteires du yuhup makú, langue de l'amazonie colombienne: morphologie et syntaxe. Tesis de doctorado. Universidad de París VII. 
Rojas, Tulio. (1998). La lengua Páez. Bogotá: Ministerio de Cultura.

Simone, Raffaele. (2001). Fundamentos de lingüística. Barcelona: Ariel. 